\title{
CASIMIR: a submillimeter heterodyne spectrometer for SOFIA
}

\section{Michael L. Edgar, Jonas Zmuidzinas}

Michael L. Edgar, Jonas Zmuidzinas, "CASIMIR: a submillimeter heterodyne spectrometer for SOFIA," Proc. SPIE 4014, Airborne Telescope Systems, (20 June 2000); doi: 10.1117/12.389129 


\title{
CASIMIR: A Submillimeter Heterodyne Spectrometer for SOFIA
}

\author{
Michael L. Edgar and Jonas Zmuidzinas \\ Submillimeter Astrophysics Group \\ California Institute of Technology \\ MC 320-47, Pasadena, CA 91125, USA.
}

\begin{abstract}
The CAltech Submillimeter Interstellar Medium Investigations Receiver (CASIMIR) is a multichannel, heterodyne spectrometer being developed for the Stratospheric Observatory for Infrared Astronomy (SOFIA). It has a very high resolution, up to a million, over the submillimeter and far-infrared wavelength range of 150 to $600 \mu \mathrm{m}$, or 2.0 to $0.5 \mathrm{THz}$. CASIMIR is extremely well suited to the investigation of both the galactic and extragalactic warm, approximately $100 \mathrm{~K}$, interstellar medium. A combination of advanced SIS and Hot Electron Bolometers (HEB) receivers will be used to cover this frequency range with very high sensitivity. CASIMIR will use only solid state local oscillators, with quasioptical coupling to the mixers. We present a description of the instrument and its capabilities, including detailed discussions of the receivers, local oscillators and IF systems.
\end{abstract}

Keywords: submillimeter, spectrometer, SOFIA, SIS, HEB

\section{INTRODUCTION}

The CAltech Submillimeter Interstellar Medium Investigations Receiver (CASIMIR) is a multichannel, far-infrared (FIR) and submillimeter heterodyne spectrometer being developed for the Stratospheric Observatory for Infrared Astronomy (SOFIA), as a first generation, PI-class instrument. It will cover the frequency range of 0.5 to $2.0 \mathrm{THz}$ with a resolution of $\delta \nu / \nu \approx 10^{6}$.

The FIR/submm is extremely important for the investigation of both the galactic and extragalactic warm $(T \approx 100 \mathrm{~K})$, interstellar medium. This material is heated by shock waves or UV radiation, phenomena that are often associated with star formation or other high energy events, e.g. supernovae or active galactic nuclei. This excited material then re-emits either as dust continuum radiation or gas line emission.

CASIMIR will be able to utilize recent advances in the sensitivity of superconducting mixers to study the fundamental rotational transitions of many astronomically significant hydride molecules, which cannot be observed with ground based telescopes. A partial list of these lines is shown in Table 1.

CASIMIR will be used to investigate the abundance and excitation of interstellar water using a number of transitions of $\mathrm{H}_{2}{ }^{18} \mathrm{O}$. While oxygen is the third most abundant element, its chemistry in interstellar clouds is poorly understood, since the atmosphere is opaque to many of its key species, such as $\mathrm{O}, \mathrm{O}_{2}, \mathrm{H}_{2} \mathrm{O}, \mathrm{H}_{3} \mathrm{O}^{+}$and $\mathrm{OH}$. Gas phase water also has an important role in the energy balance of molecular clouds due to radiative cooling or heating through FIR/submm rotational transitions. ${ }^{1}$ Figure 1 shows the rotational energy levels for $\mathrm{H}_{2}{ }^{18} \mathrm{O}$, indicating the large number of low excitation level transitions visible to CASIMIR. Figure 2 shows results from observations of the $547 \mathrm{GHz} \mathrm{H}_{2}{ }^{18} \mathrm{O}$ line obtained on the Kuiper Airborne Observatory (KAO) and predictions of the line intensities that will be obtained for the same source with CASIMIR on SOFIA.

The $\mathrm{H}_{2} \mathrm{D}^{+}$ion is of particular interest since it is the deuterated version of $\mathrm{H}_{3}^{+}$, which is believed to be responsible for driving much of the chemistry of molecular clouds. Numerous ground-based searches have been made using the more highly excited transitions of $\mathrm{H}_{2} \mathrm{D}^{+}$, and the $372 \mathrm{GHz}$ line has now been detected in one object. ${ }^{2}$ However, this line traces hot, dense gas, in which the chemistry is more complicated and the $\mathrm{H}_{2} \mathrm{D}^{+}$abundance is expected to 


\begin{tabular}{|c|c|c|c|c|c|}
\hline \multirow[t]{2}{*}{ Species } & \multirow[t]{2}{*}{ Transition } & \multirow{2}{*}{$\begin{array}{c}\text { Frequency } \\
\text { GHz }\end{array}$} & \multirow{2}{*}{$\begin{array}{c}E_{\text {lower }} \\
\mathrm{K}\end{array}$} & \multicolumn{2}{|c|}{ Atmospheric Transmission } \\
\hline & & & & $1 \mathrm{~mm} \mathrm{H}_{2} \mathrm{O}$ & SOFIA \\
\hline $\mathrm{CH}$ & $F_{1} \rightarrow F_{2} ; J=3 / 2^{-} \rightarrow 1 / 2^{+}$ & 536.76 & 0.0 & $0 \%$ & $97 \%$ \\
\hline $\mathrm{H}_{2}{ }^{18} \mathrm{O}$ & $1_{10} \rightarrow 1_{01}$ & 547.68 & 34.2 & $0 \%$ & $81 \%$ \\
\hline $\mathrm{NH}_{3}$ & $1_{0} \rightarrow 0_{0}$ & 572.50 & 0.0 & $0 \%$ & $94 \%$ \\
\hline $\mathrm{H}_{2}{ }^{18} \mathrm{O}$ & $2_{11} \rightarrow 2_{02}$ & 745.32 & 100.6 & $0 \%$ & $82 \%$ \\
\hline $\mathrm{NH}$ & $N=1 \rightarrow 0 ; J=2 \rightarrow 1$ & 974.48 & 0.0 & $0 \%$ & $96 \%$ \\
\hline $\mathrm{H}_{3} \mathrm{O}^{+}$ & $0_{0}^{-} \rightarrow 1_{0}^{+}$ & 984.66 & 7.5 & $0 \%$ & $65 \%$ \\
\hline $\mathrm{NH}^{+}$ & $3 / 2^{+} \rightarrow 1 / 2^{-}$ & 998.90 & 0.0 & $0 \%$ & $95 \%$ \\
\hline $\mathrm{HF}$ & $1 \rightarrow 0$ & 1232.48 & 0.0 & $0 \%$ & $30 \%$ \\
\hline $\mathrm{H}_{2} \mathrm{D}^{+}$ & $1_{01} \rightarrow 0_{00}$ & 1370.09 & 0.0 & $0 \%$ & $94 \%$ \\
\hline $\mathrm{N}^{+}$ & ${ }^{3} P J=1 \rightarrow 0$ & 1461.13 & 0.0 & $0 \%$ & $92 \%$ \\
\hline${ }^{16} \mathrm{OH}$ & ${ }^{2} \Pi_{1 / 2} J=3 / 2^{+} \rightarrow 1 / 2^{-}$ & 1837.82 & 181.9 & $0 \%$ & $94 \%$ \\
\hline $\mathrm{C}^{+}$ & ${ }^{2} P J=3 / 2 \rightarrow 1 / 2$ & 1900.54 & 0.0 & $0 \%$ & $88 \%$ \\
\hline $\mathrm{CH}_{2}$ & $1_{10} \rightarrow 1_{01}$ & 1917.66 & 22.4 & $0 \%$ & $99 \%$ \\
\hline $\mathrm{CO}$ & $18 \rightarrow 17$ & 1956.02 & 751.7 & $0 \%$ & $90 \%$ \\
\hline
\end{tabular}

Table 1. Selected lines within CASIMIR's frequency range. The table also compares the atmospheric transmission at these frequencies for typical conditions at Mauna Kea with those at SOFIA's operating altitude.

be low. The $1370 \mathrm{GHz}$ ground-state line is a better choice. The only previous search for this line was made towards Orion, using the $\mathrm{KAO}$, and resulted only in a tentative detection of an absorption feature. ${ }^{3}$

Another transition of major importance is the $1461 \mathrm{GHz}$ transition of the nitrogen ion, $\mathrm{N}^{+}$, which traces the warm ionized interstellar medium. COBE has shown ${ }^{4}$ that the two fine-structure lines of $\mathrm{N}^{+}$are the brightest emitted by our galaxy, apart from $1900 \mathrm{GHz} \mathrm{C}^{+}$line. The high-J lines of $\mathrm{CO}$ will also be accessible to CASIMIR. These lines mainly trace shocked gas and are well known from work with the KAO, including high-resolution heterodyne spectroscopy. ${ }^{5}$

\section{INSTRUMENT CONFIGURATION}

Figure 3 shows a block diagram and the signal path for the CASIMIR instrument. The light passes through an optics assembly directing it into one of two cryostats, where it is quasioptically coupled to a mixer. There it is combined with the local oscillator signal (LO) which is also quasioptically coupled. The resultant intermediate frequency (IF) signal, $4-8 \mathrm{GHz}$, is amplified by a HEMT amplifier on the cryostat cold work surface and then by a room temperature amplifier. After this, the IF is downconverted to one of the frequencies shown in the figure and fed to one of three microwave spectrometers. These subsystems are discussed in more detail below.

The instrument is controlled by the Command and Data Handling subsystem (CDHS). This is a VME based system which interfaces to a combination of several custom and commercial off-the-shelf electronics systems for controlling and monitoring the various instrument functions. The VME system also contains a DSP which will be used for controlling the chopping secondary mirror and providing timing signals for the microwave spectrometers. Communications between the various instrument subsystems will be via a combination of the IEEE- 488 bus and ethernet connections.

A general purpose computer for control of the instrument will be located at one of the operators' positions. This will be the primary control point for the instrument. In most cases, communications between the operators and the instrument is expected to be solely through a fibre optic fast ethernet connection, with some coaxial signal lines for diagnostic purposes. Communications to the observatory itself will be through an ethernet connection, apart from signals to and from the secondary mirror. 


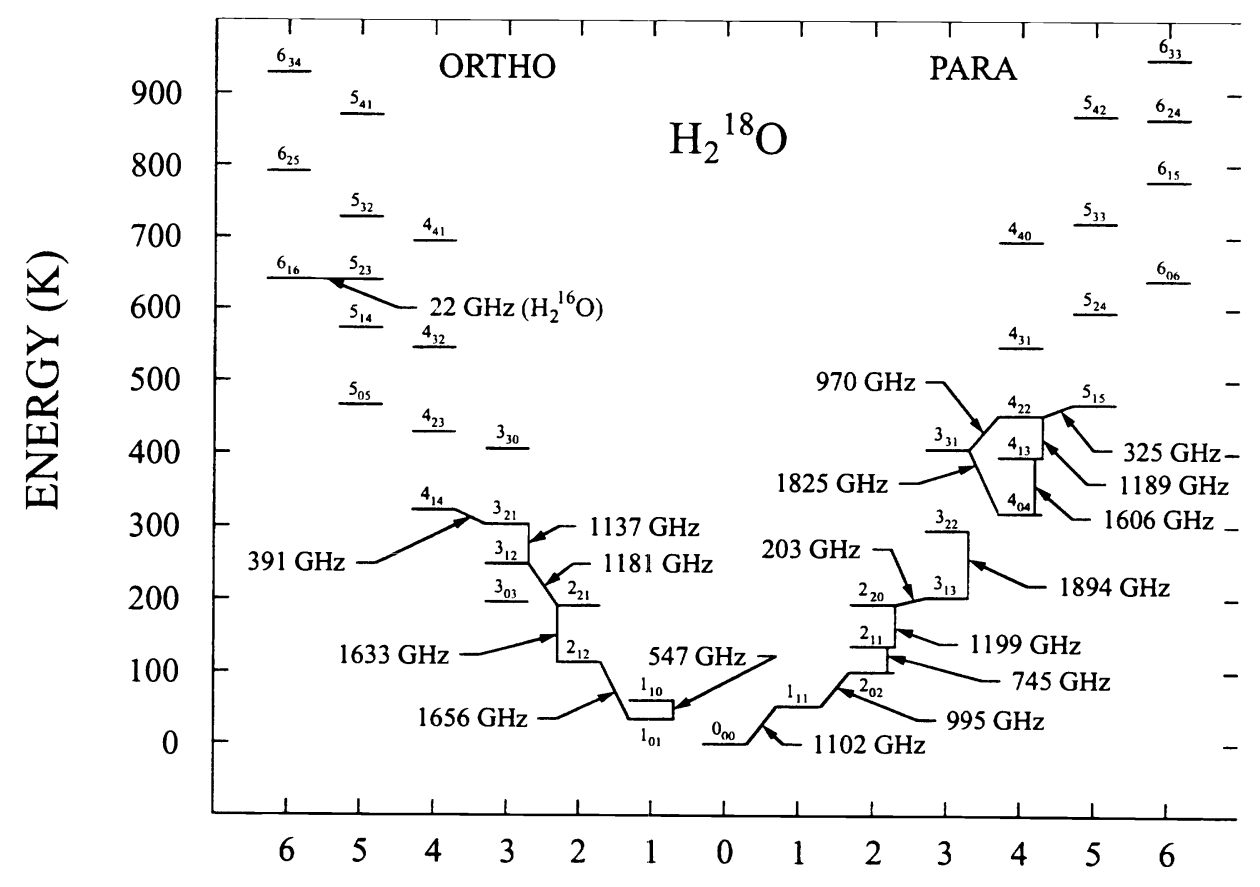

ANGULAR MOMENTUM, $J$

Figure 1. Rotational energy levels of the $\mathrm{H}_{2}{ }^{18} \mathrm{O}$ molecule.
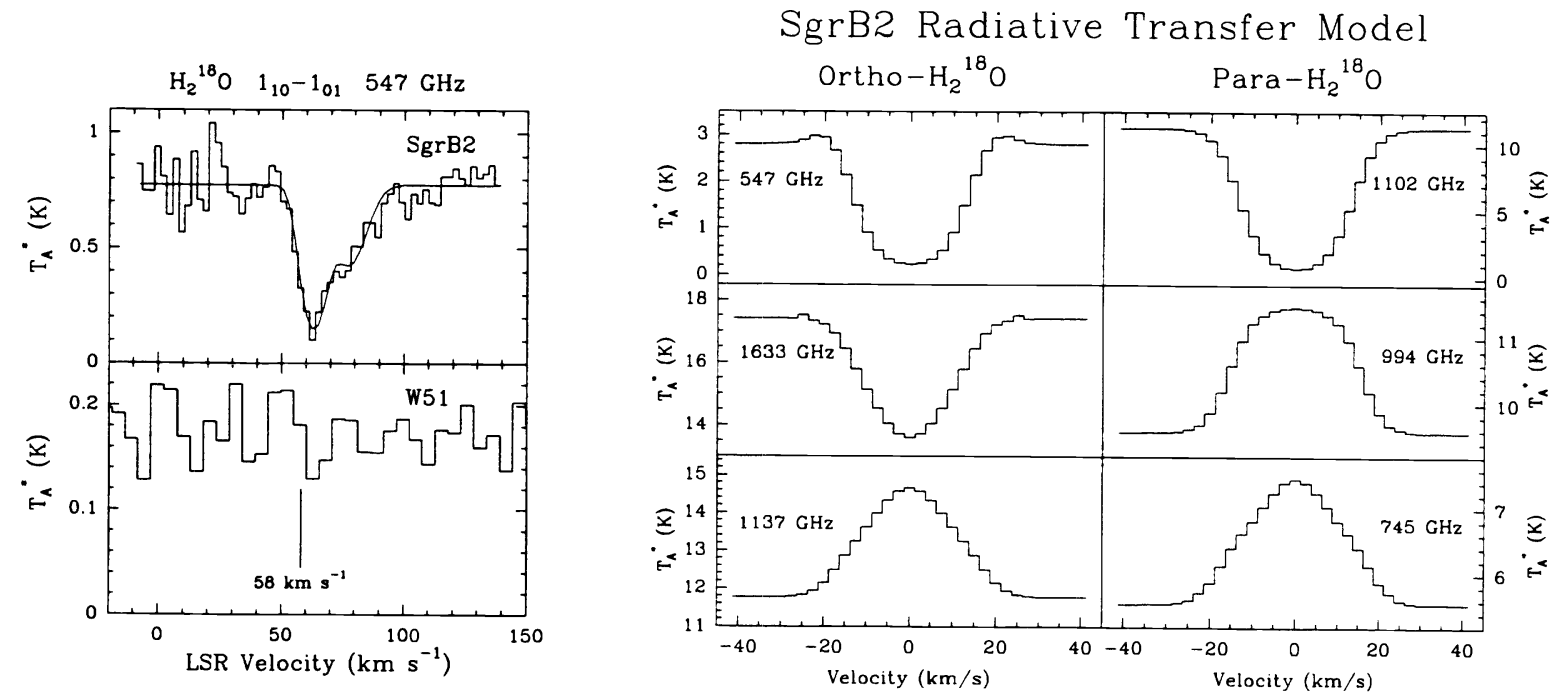

Figure 2. Left: $\mathrm{KAO}$ observations of $\mathrm{H}_{2}{ }^{18} \mathrm{O}$ at $547 \mathrm{GHz}$. Right: Predicted $\mathrm{H}_{2}{ }^{18} \mathrm{O} \mathrm{SgrB} 2$ line intensities for SOFIA, using a spherical model $\left(n_{\mathrm{H} 2}(r) \sim r^{-2}\right.$ and $\left.T(r) \sim r^{-0.5}\right)$ which matches existing CO, dust, and $\mathrm{H}_{2}{ }^{18} \mathrm{O}$ data. 


\subsection{Structure}

Figure 4 shows the mechanical configuration of the instrument. The structure has three main elements: a mounting flange, the electronics frame and the optics box. The mounting flange is bolted directly to the instrument flange of the telescope. This flange provides the only mechanical support for the optics box and the electronics frame. The optics box and the frame are mounted separately to this mounting flange and are not in physical contact with each other.

The optics box contains almost all of the optics necessary to relay the FIR/submm signal to the mixers, as well as an optical boresight camera. There are no optical windows between the optics box and the telescope. Therefore, the box's interior is exposed directly to the telescope cavity and the internal air pressure is similar to that outside the aircraft. While the optics box is a pressure vessel, it is not cryogenically cooled. The optics box also provides the mechanical mounts for the two cryostats. There are no mechanical or motion feedthroughs on the optics box, only electrical feedthroughs.

The electronics frame is essentially two standard 19 inch racks approximately $800 \mathrm{~mm}$ high, mounted side by side. Approximately $120 \mathrm{~kg}$ of electronics will be mounted within the frame in standard 19 inch rack bins. The frame will contain almost all of the electrical systems necessary for the operation of CASIMIR, including the CDHS.

Only a few of CASIMIR's electrical systems are not mounted within the electronics frame. Two of the microwave spectrometers, with a combined mass of approximately $100 \mathrm{~kg}$, will be mounted in the electronics rack located on the telescope's counterweight. Therefore, all of the microwave cables will be short and all the IF components will rotate with the telescope, reducing problems due to signal losses in the cables and noise caused by cable motions.

\subsection{Optics}

The optical system consists of three major components: an optical boresight camera, a FIR/submm relay system and a FIR/submm calibration system. Figure 5 shows a schematic diagram of the optics contained within the optics box. A plane mirror mounted on a precision rotary stage has six predetermined positions. One position directs the incoming telescope beam into the boresight, two positions direct that beam to either of the cryostats, two positions direct the beam from the calibration source to either cryostat and a final position directs an alignment laser onto the boresight. Only one cryostat or the boresight can be illuminated at any one time. The field of view at the telescope's prime focus has a diameter of 8 arcmin and the optics within the optics box are large enough to image the full field. The beam direction mirror will also be mounted on a tilt stage, to allow for the correction of any small misalignments between components.

The relay optics are essentially identical for each cryostat. The incoming beam from the telescope is directed to one of the off-axis elliptical mirrors. This $\mathrm{f} / 19$ beam has already passed through its primary focus and the elliptical mirror reflects the beam through an angle of $90^{\circ}$ and converts it to a converging $\mathrm{f} / 10$ beam, which enters the cryostat through a window in its base. This window is the only warm transmissive element in the entire beam path. There will be up to two mixers in each cryostat, each of which will have a second off-axis elliptical mirror located on the cold-work surface. This second mirror will also reflect the beam through $90^{\circ}$ and convert it from $\mathrm{f} / 10$ to about $\mathrm{f} / 4.5$. Finally a silicon lens assembly, as seen in Figure 6, will match the beam to the mixer's antenna.

The calibration subsystem is used to illuminate the receivers alternately with a hot or cold load. The cold load will be at the ambient temperature within the optics box and the hot load will be approximately $100^{\circ} \mathrm{C}$ higher. The temperatures of both loads will be constantly monitored by the CDHS and the hot load temperature will be maintained at its setpoint by a feedback-controlled heater. The loads are selected by intermittent movement of a rotary stage to place either load in the beam path. The beam is folded through $90^{\circ}$ by the FIR plane mirror, as shown in Figure 5. It is then directed to one of the elliptical mirrors by the beam direction mirror. The beam is chopped by a rotary chopper blade, rotating continuously at a speed between 0.5 and $5 \mathrm{~Hz}$. A $1 \mathrm{~mW}$ semiconductor laser is placed underneath the loads. When the loads are rotated out of the beam path, the laser beam can be directed by the beam direction mirror to the boresight, for alignment purposes.

The CASIMIR instrument will be used with a fully reflective tertiary mirror, rather than a dichroic. Therefore, a separate optical boresight camera is required for beam finding, etc. The beam direction mirror diverts the beam into the boresight, which consists of a fairly conventional lens assembly of commercial off-the-shelf optics and a folded beam path. The camera consists of a conventional $1024 \times 1024,9 \mu \mathrm{m}$ square pixel CCD covering a $6 \times 6$ arcmin field of view. The CCD camera is located actually inside the optics box, with a linear translation stage to provide a variable focus. 


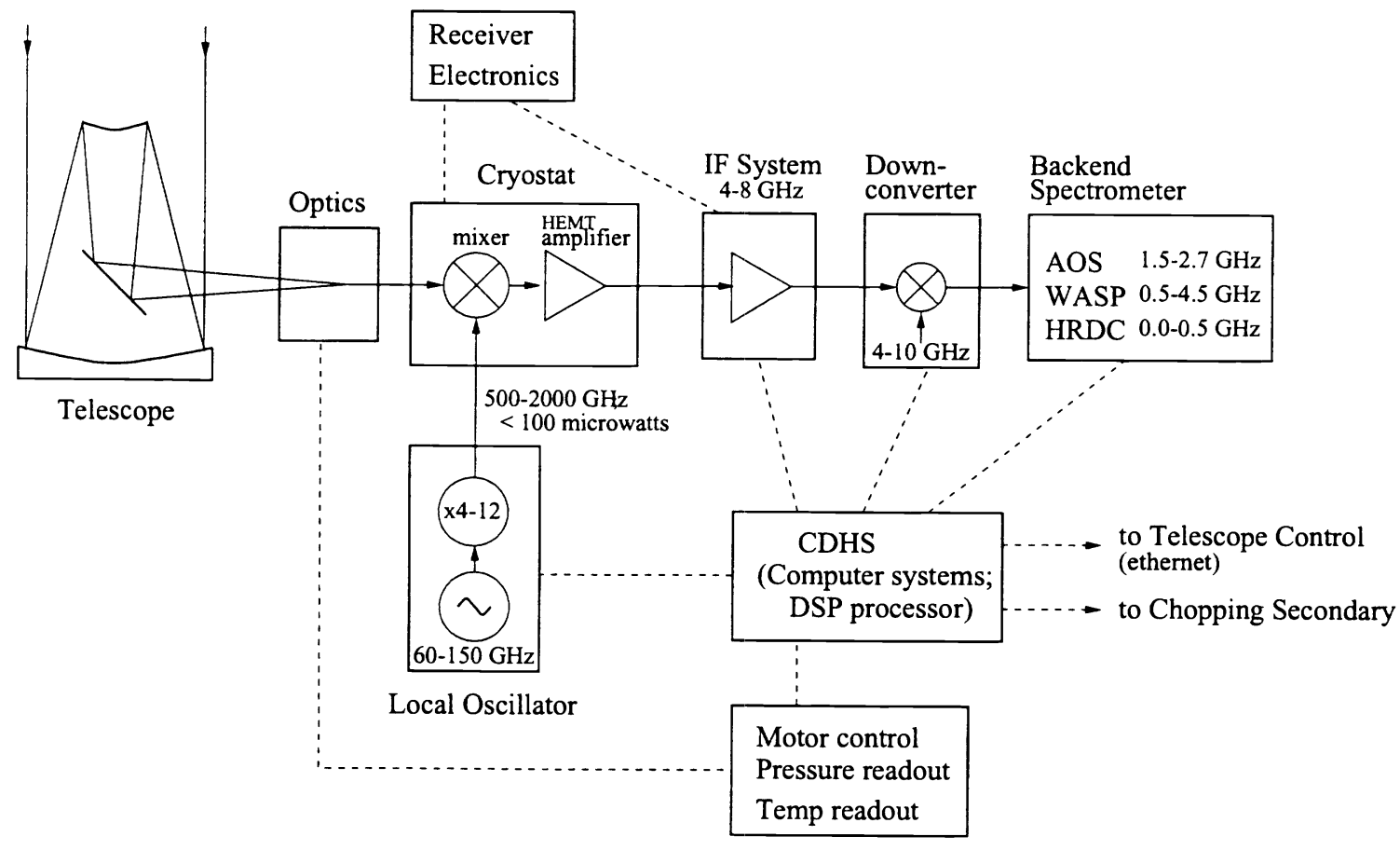

Figure 3. Block diagram and signal path for the CASIMIR instrument.

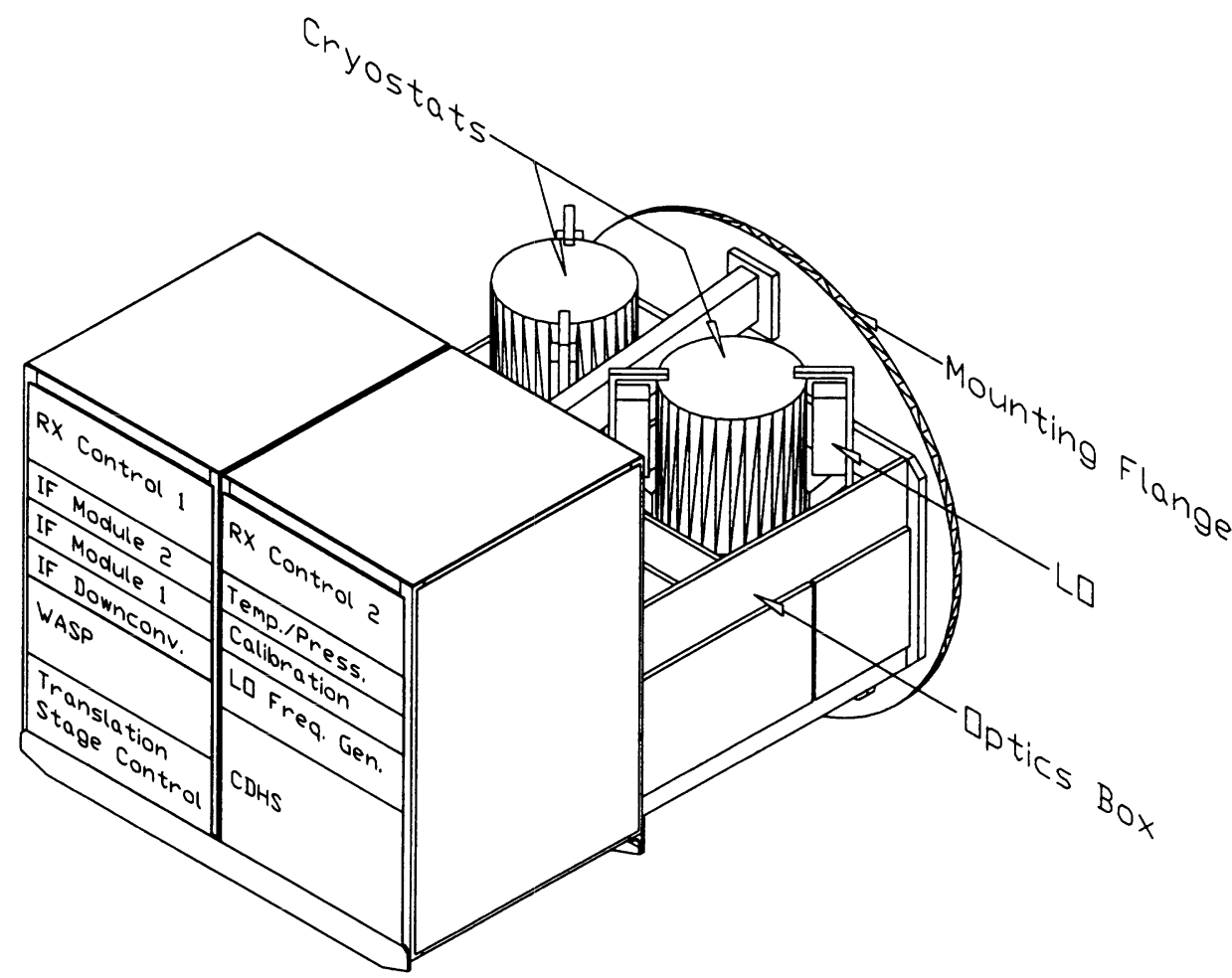

Figure 4. Schematic diagram of the mechanical configuration of the CASIMIR instrument. Apart from the cryostats, the structure is fabricated from Al-6061. The approximate dimensions are $1 \mathrm{~m}$ wide by $1 \mathrm{~m}$ high by $1.4 \mathrm{~m}$, long with a mass of approximately $450 \mathrm{~kg}$. The view is towards the telescope in the aircraft rear. 


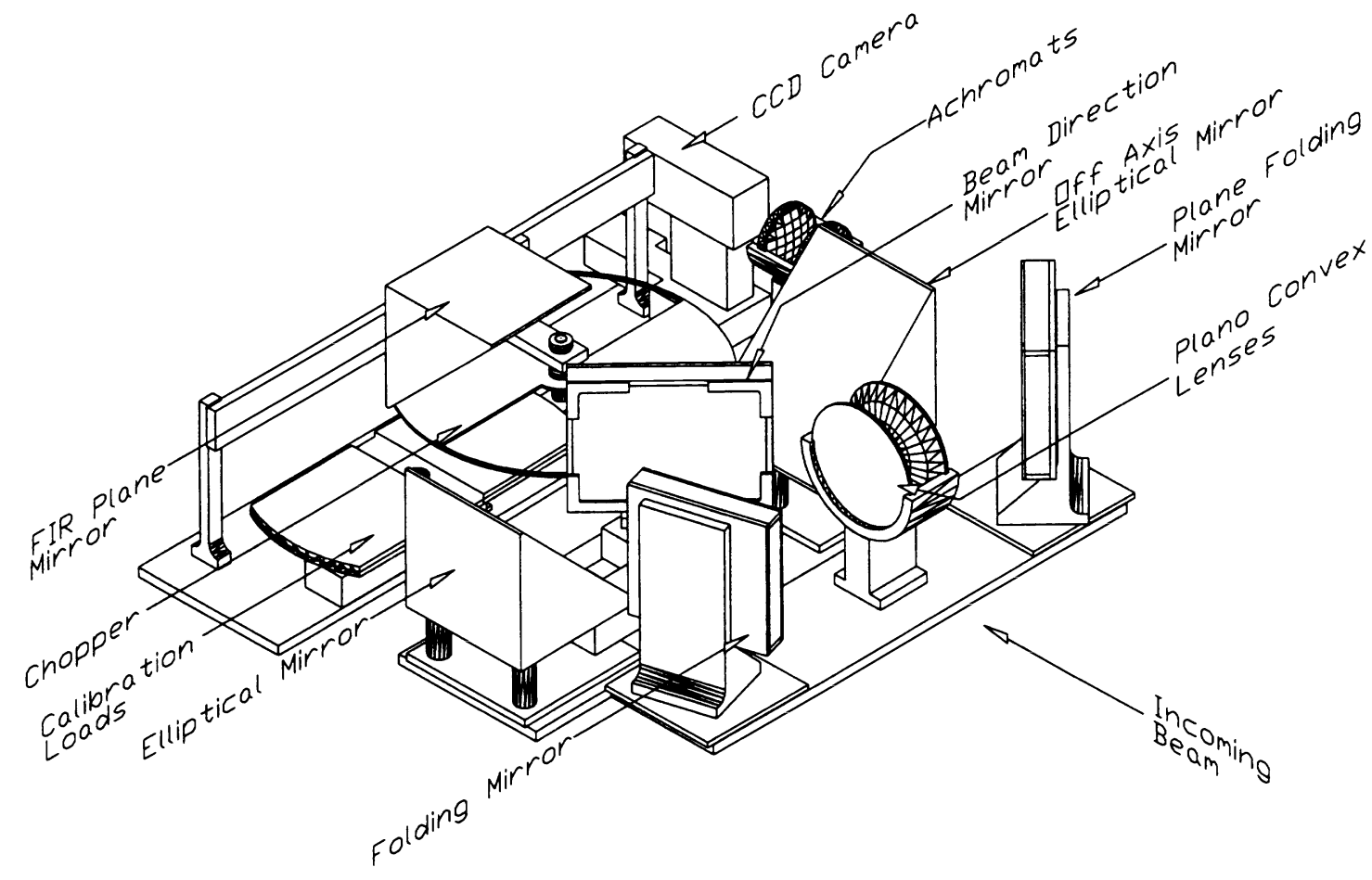

Figure 5. Schematic diagram of the optics. The diagram shows the optical arrangement of the instrument but does not include details of the proposed optomechanics for the flight instrument. Typical dimensions of the large optics are of the order of $150 \mathrm{~mm}$ square. The individual components are discussed in the text.

The CDHS constantly monitors the pressures and temperatures within the optics box and cryostats. It also directly controls the hot load temperature, all motions and the CCD camera.

\subsection{Cryostats}

The cryostats used on CASIMIR are small and of conventional design. They will be approximately $300 \mathrm{~mm}$ diameter and $500 \mathrm{~mm}$ high, containing approximately 3 litres each of liquid nitrogen and helium. The mounting of the cryostats will provide a pressure seal between the the baseplate of the cryostat and the top of the optics box.

\section{RECEIVERS}

CASIMIR will use two kinds of superconducting detectors to cover the 0.5 to $2.0 \mathrm{THz}$ bandwidth in 7 bands. Tunneljunction (SIS) mixers will be used to cover the $0.5-1.2 \mathrm{THz}$ range in four bands, while hot-electron bolometers (HEB) will be used at higher frequencies. On any given flight, there will be two cryostats mounted on the instrument, with up to 2 mixers in each, giving a maximum of 4 receiver bands available for each flight.

\subsection{SIS}

Figure 6 shows the quasioptical mixer design. A planar, twin slot antenna ${ }^{6}$ is lithographically fabricated on a silicon substrate along with the SIS junctions. This chip is then placed behind an antireflection coated, silicon, hyperhemispherical lens which focuses the incoming radiation onto the antenna.

The sensitivity of SIS mixers can, in theory, approach the quantum limit, $T_{N}=h \nu / k_{B} \approx 48 \mathrm{~K}$ at $1 \mathrm{THz}$. However, in practice, below the gap frequency of niobium, $700 \mathrm{GHz}$, the sensitivity limit is $2-5$ times the quantum limit. Above $700 \mathrm{GHz}$, the photon energy is high enough to break Cooper pairs, which makes the niobium tuning circuits very lossy, severely reducing sensitivity. 

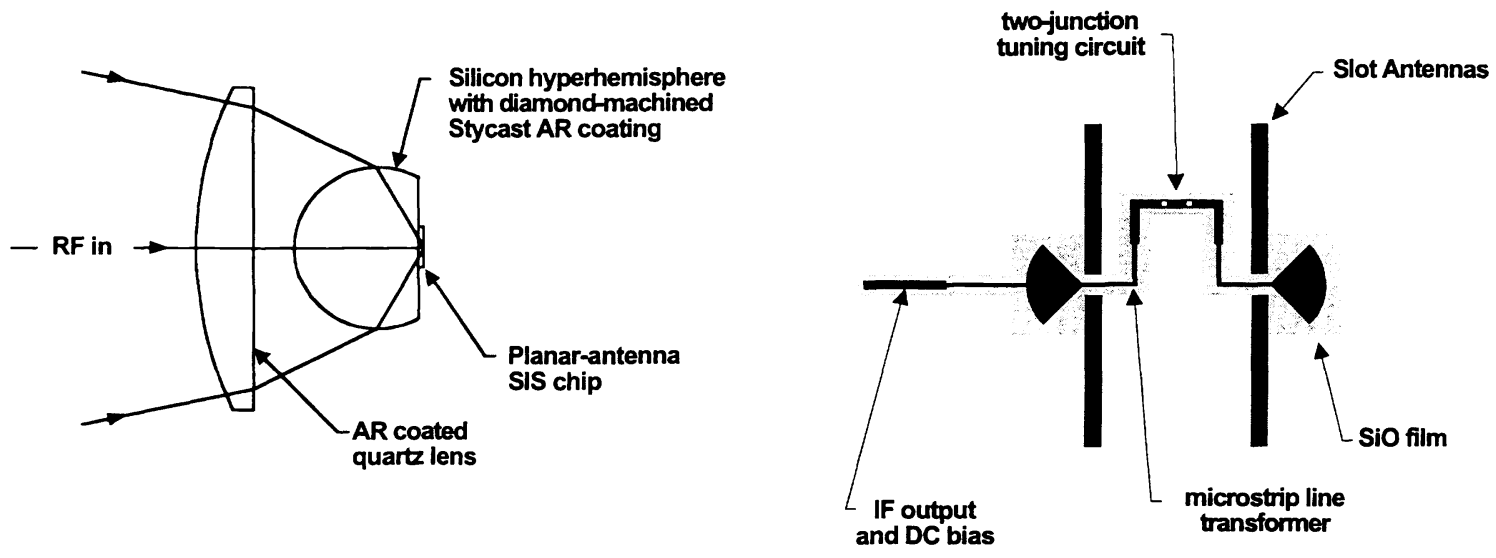

Figure 6. Schematic of the quasioptical mixer design. Left: the optical configuration of the mixer. Right: the SIS chip consists of a twin slot antenna, a microstrip transformer and a tuning circuit containing two SIS junctions.

SIS mixers can be extended to frequencies above $700 \mathrm{GHz}$ by using materials with higher gap frequencies. NbTiN has a gap frequency approximately 1.7 times higher than niobium, and so may be useful in mixers up to $1.2 \mathrm{THz}^{7}{ }^{8}$ Figure 7 shows results recently obtained at the Caltech Submillimeter Observatory (CSO), ${ }^{9}$ with an SIS mixer with low loss NbTiN films. The noise temperature for the mixer is $205 \mathrm{~K}$ (DSB) at $800 \mathrm{GHz}$, which is an improvement of better than a factor of 2 over the previous results at this frequency.

The current density of the SIS junctions can be increased by at least a factor of 3 by replacing the standard aluminum oxide barrier with aluminum nitride (AlN). These higher current junctions are less susceptible to losses in the tuning circuit and the mixer bandwidths can be increased from $\approx 100 \mathrm{GHz}$ to $\approx 300 \mathrm{GHz} .{ }^{10}$ An example of the response of this type of mixer is shown in Figure 8. The heterodyne noise temperatures of the junctions with the high current density AlN barriers have been found to be equivalent to the those with lower current density Al-oxide barriers.

\subsection{HEB}

For frequencies beyond those accessible with SIS mixers, we plan to use diffusion-cooled HEB mixers. The same quasioptical planar antenna technique used for the SIS mixers is used for the HEBs. The HEB device is an ultrathin niobium film fabricated with e-beam lithography into a submicron bridge, connected to normal metal pads at either end, which serve as heatsinks. ${ }^{11}$ The small heat capacity, due to the submicron size, and the high thermal conductance, due to cooling by electron diffusion, lead to very fast response times. Times of the order tens of picoseconds have been measured for microbridges $<0.5 \mu \mathrm{m}$ long by $0.1 \mu \mathrm{m}$ wide by $10 \mathrm{~nm}$ thick. The short thermal response time allows HEBs to be used as heterodyne mixers with IFs up to $6-10 \mathrm{GHz} .{ }^{12}$ Sensitive HEB mixers have been operated up to $2.5 \mathrm{THz} .{ }^{13}$

\subsection{Receiver Control}

CASIMIR will require numerous custom circuits for operating the receivers. Separate biasing circuits are required for the mixers, the cryogenic IF amplifiers and the LO multipliers. Circuits are also required for controlling and monitoring the IF system and controlling the current in a magnet coil, necessary to suppress Josephson currents in the SIS mixers.

CASIMIR will use a fully automated receiver control system which is being developed for the CSO. This consists of 5 hybrid analog-digital cards, one for each of the major functions described above. Each of the cards has an FPGA digital interface to a common, custom data and power bus. Communications with the individual cards is carried out via an ethernet connection to a single board computer on the bus. Each set of 6 cards can control 2 mixers. The power supplies, bus and cards are all contained within a single $4 \mathrm{U}, 19$ inch rack bin. By using two of these systems, we will be able to have full computer control of all 4 of CASIMIR's receiver bands, via ethernet from any workstation on the aircraft. 


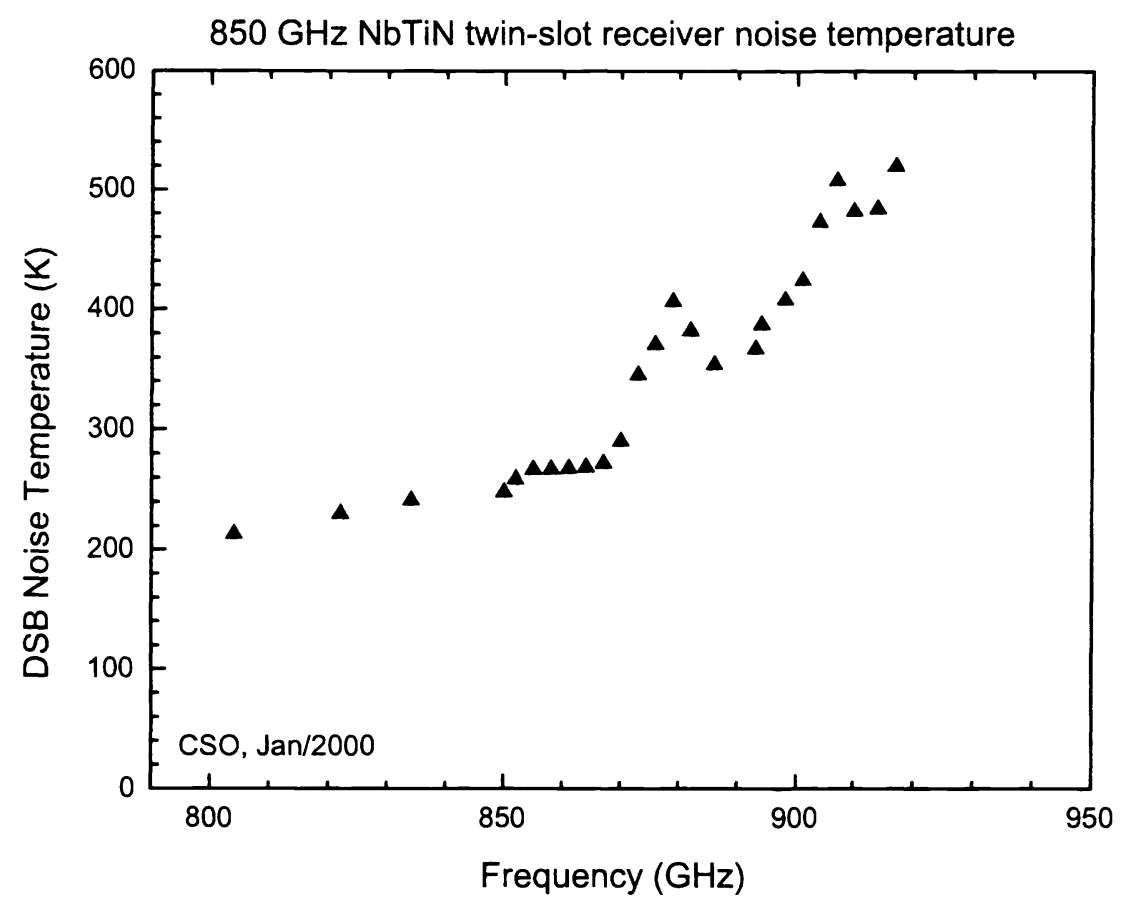

Figure 7. Receiver noise temperatures measured at the Caltech Submillimeter Observatory. The mixer is a twin-slot device using NbTiN ground plane and wiring layers, and a $\mathrm{Nb} / \mathrm{AlN} / \mathrm{NbTiN}$ junction with a current density around $10 \mathrm{kA} \mathrm{cm}^{-2}$. The increase of noise temperature with frequency above $850 \mathrm{GHz}$ is consistent with the direct-detection frequency response measured with our Fourier transform spectrometer (FTS).

$\mathrm{Nb} / \mathrm{AlN} / \mathrm{Nb}$ junction with $\mathrm{Nb}$ tuning circuit (device 62)

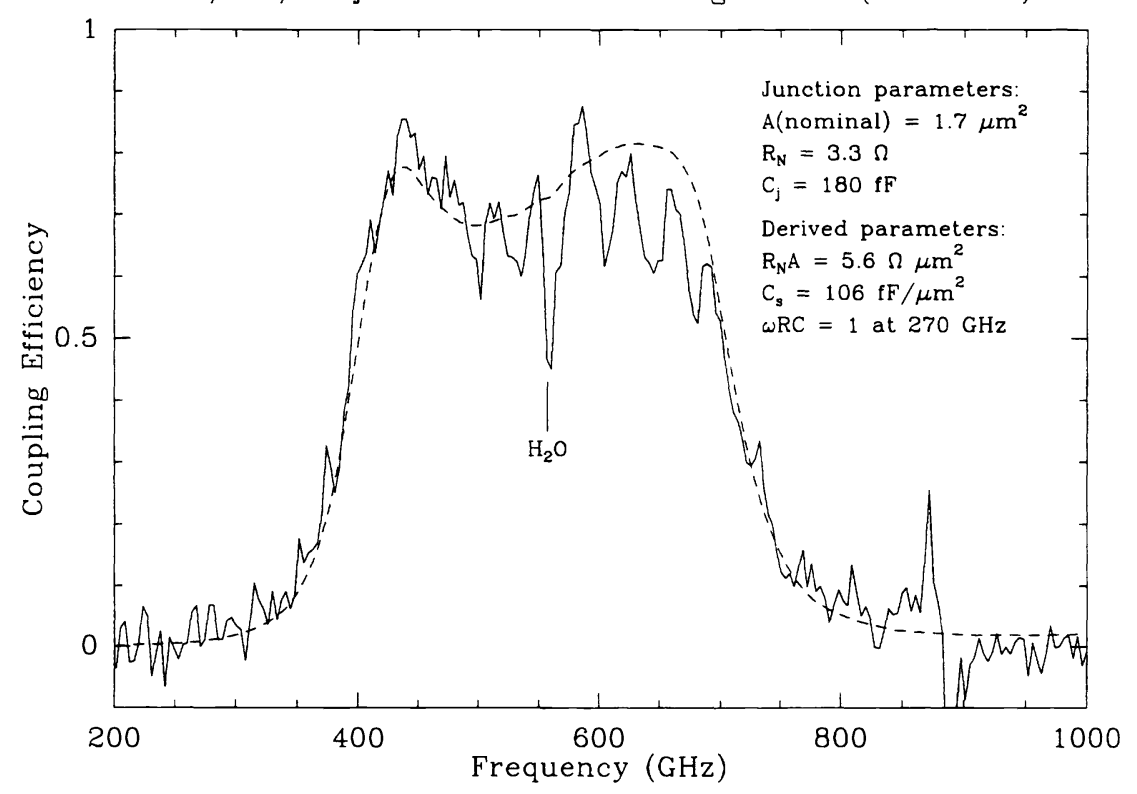

Figure 8. The measured (solid line) and predicted (dashed) direct-detection response of a twin slot SIS mixer with high current density $\left(\sim 35 \mathrm{kA} \mathrm{cm}^{-2}\right) \mathrm{Nb} / \mathrm{AlN} / \mathrm{Nb}$ junctions, showing the very wide tuning bandwidth (300 GHz). The peak coupling efficiency is only $\sim 70 \%$ because the tuning circuit was designed for $10 \mathrm{kA} \mathrm{cm}^{-2}$ junctions. 


\section{LOCAL OSCILLATORS}

The LO system consists of a microwave frequency synthesizer controlled by the CDHS. The microwave signal will drive an active multiplier chain followed by a millimeter-wave HEMT power amplifier (the millimeter-wave source, MWS). There will be a separate MWS for each mixer, so there will be up to 2 per cryostat, mounted on the sides of the cryostats as shown in Figure 4.

The output from each MWS will be coupled to a dedicated frequency multiplier (FM) via waveguide. These FM's will use planar MMIC doubler and tripler chips mounted in waveguide blocks, and will multiply the frequency of the MWS by a factor of from 4 to 12 , in order to provide the LO signal at $0.5-2 \mathrm{THz}$. The output of the FM is emitted by a feedhorn. This divergent beam is reflected through $90^{\circ}$ and converted into an $\mathrm{f} / 10$ beam by an off-axis elliptical mirror located on the side of the cryostat, directly below the FM feedhorn. The beam passes through a window in the cryostat wall to a mylar beamsplitter directly below the receiver elliptical mirror on the cold work surface. The beamsplitter directs a portion of the LO signal power onto the receiver elliptical mirror, combining it with the incoming, astronomical signal.

\section{INTERMEDIATE FREQUENCY SYSTEM}

CASIMIR will use a $4-8 \mathrm{GHz}$ intermediate frequency (IF) band. The $4 \mathrm{GHz}$ bandwidth is necessary to allow observations of galaxies at the short submillimeter wavelengths. Numerous IF components are being developed with special attention being paid to gain flatness.

\subsection{Cryogenic HEMT IF Amplifiers}

A 4-8 GHz cryogenic low noise amplifier has been designed and fabricated using Quasi-Monolithic Microwave Integrated Circuit (QMMIC) technology. The QMMIC consists of a thin-film passive circuit on a GaAs substrate with three $160 \mu \mathrm{m}$ gate InP HEMTs bump-bonded to it. Figure 9 shows the size and configuration of the amplifier's mounting block and Figure 10 shows the predicted gain of the amplifier compared to preliminary measurements made at $77 \mathrm{~K}$. At $4 \mathrm{~K}$, the amplifier drew a total of only $8 \mathrm{~mW}$ bias power and the noise temperature varied smoothly between $6 \mathrm{~K}$ and $10 \mathrm{~K}$. The amplifier will be used with an isolator (see below).

\subsection{Cryogenic Isolator}

Cryogenic isolators with a 4-8 GHz bandpass are now commercially available from Pamtech Inc., ${ }^{14}$ as a result of a development contract for the FIRST/HIFI instrument. ${ }^{15}$

\subsection{Room Temperature IF Amplifier Module}

The room temperature IF electronics consist of a 4-8 GHz amplifier module. This is an integrated unit developed by contract to CTT Inc. ${ }^{16}$ containing a low-noise amplifier, a voltage-variable attenuator, band-defining filter, power amplifier, a directional coupler for monitoring the IF power level, and a switch for setting the IF power zero level. An isolator at the input of the module minimizes standing wave ripples between the cryostat and amplifier module. The gain of the unit is $65 \mathrm{~dB}$. Figure 11 shows the better than $\pm 1 \mathrm{~dB}$ gain flatness of the unit with the internal voltage-variable attenuator(VVA) set to $-10 \mathrm{~dB}$. The noise temperature of the unit is typically $300 \mathrm{~K}$. A diode is connected to the monitor port for measuring the signal strength and adjusting the VVA to prevent the internal amplifiers from saturating.

\section{MICROWAVE SPECTROMETERS}

We expect to have three separate types of microwave spectrometers for analyzing the IF signal, each capable of processing the full $4 \mathrm{GHz}$ bandwidth. A dedicated IF downconverter module will be developed for each type of spectrometer to convert the IF signal into the necessary frequency bands. Each of the microwave spectrometers will have their own embedded processors and will be essentially independent subsystems. Timing and synchronization signals between the CDHS and all of the spectrometers will be provided by direct connections. All other communications will be via ethernet. Therefore, we expect to be able to easily reconfigure CASIMIR to use any combination of these spectrometers. We expect that all three spectrometers could be available on any given flight. 


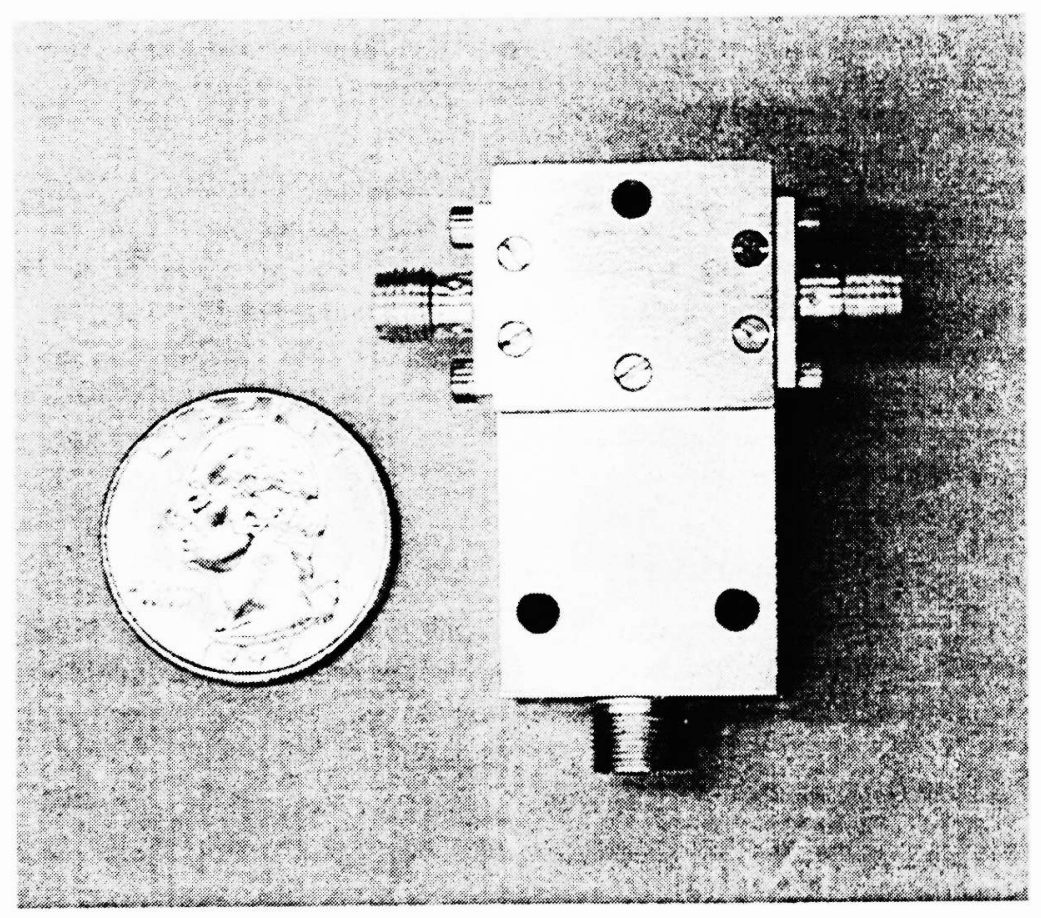

Figure 9. The cryogenic HEMT IF amplifier block.

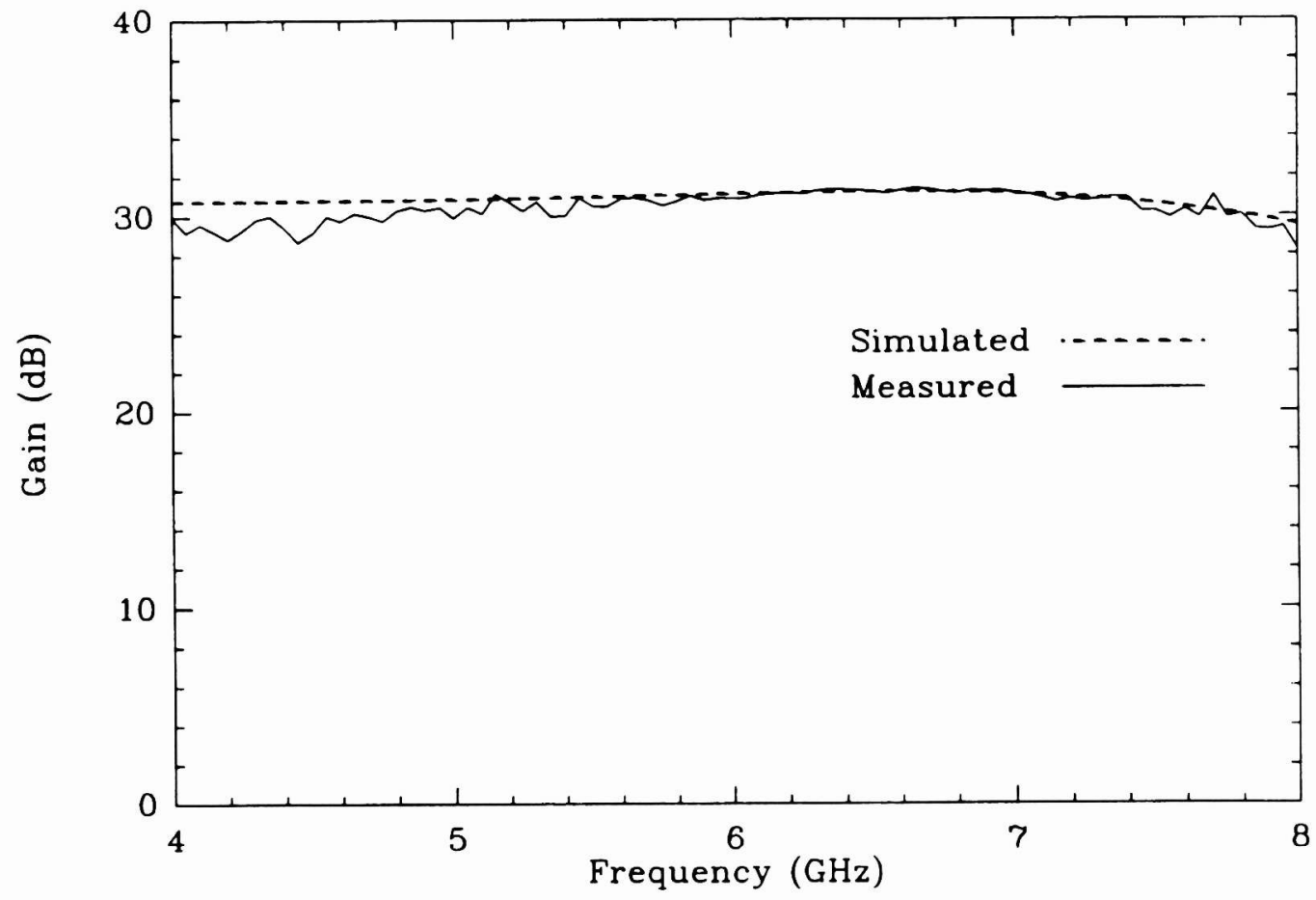

Figure 10. Predicted gain of the cryogenic HEMT IF amplifier compared to preliminary measurements at $77 \mathrm{~K}$. 


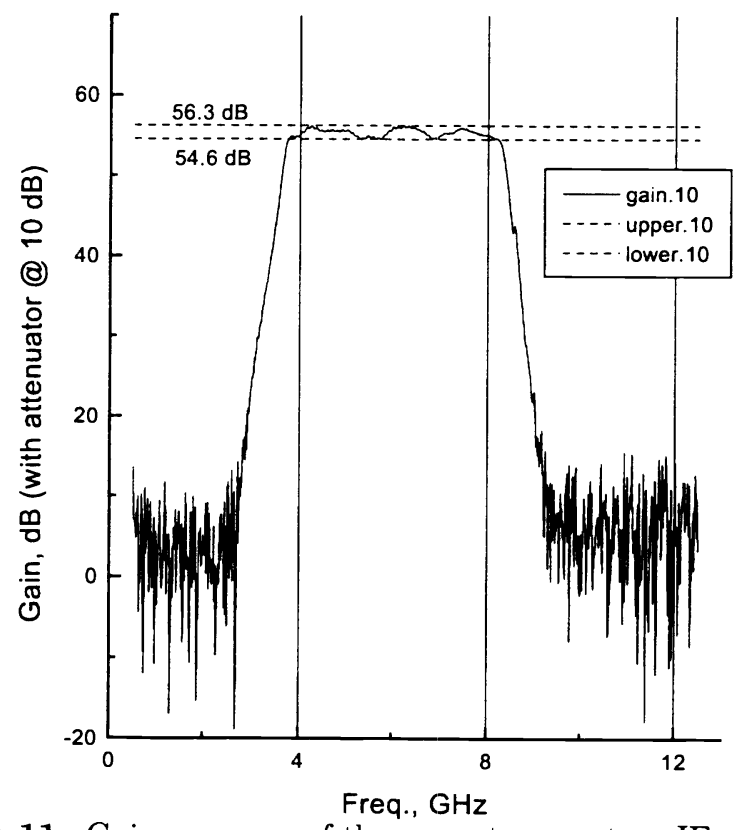

Figure 11. Gain response of the room temperature IF amplifier module.

\subsection{Acousto-Optical Spectrometer (AOS)}

The University of Köln has developed a $4 \times 1 \mathrm{GHz}$ bandwidth array $\mathrm{AOS}^{17}$ which will be used on another submillimeter heterodyne spectrometer for SOFIA, the GREAT instrument, ${ }^{18}$ as well as with CASIMIR. The spectrometer consists 4 parallel channels, each with $1 \mathrm{GHz}$ bandwidth (1.6-2.6 GHz bandpass) and $1 \mathrm{MHz}$ resolution.

\subsection{High Resolution Digital Correlator (HRDC)}

The HRDC will be based on a new digital correlator developed at Caltech's Owens Valley Radio Observatory. ${ }^{19}$ It consists of a FPGA card capable of processing a $500 \mathrm{MHz}$ analog bandwidth with $2 \mathrm{MHz}$ resolution and a separate card with two digitizers operating at $500 \mathrm{MHz}$, i.e. a total of $1 \mathrm{GSample/s.} \mathrm{These} \mathrm{cards} \mathrm{are} \mathrm{based} \mathrm{on} \mathrm{the} \mathrm{Compact-}$ PCI bus. A $4 \mathrm{GHz}$ bandwidth spectrometer would consist of 8 sets of these cards.

Varying the digitizing rate and reprogramming the FPGAs may allow much higher resolution over narrower bandwidths. It is expected that this reprogramming could take place in flight, so allowing a reconfigurable spectrometer.

\subsection{Wideband Analog SPectrometer (WASP)}

The WASP ${ }^{2021}$ is being developed at the University of Maryland. It is an analog correlator spectrometer with a $4 \mathrm{GHz}$ bandwidth and 250 channels, providing a resolution of $16 \mathrm{MHz}$. In contrast to the other spectrometer types, WASP analyzes the $4 \mathrm{GHz}$ bandwidth as a whole, without splitting the IF into subbands. It is expected that this approach will be more suitable for wide extragalactic spectral lines.

\section{ACKNOWLEDGMENTS}

Various subsystems of the CASIMIR instrument are being developed by a number of people at several institutions. At Caltech, the group includes: J. Ward (IF amplifiers), D. Miller (room-temperature IF system), J. Kawamura, A. Karpov, and J. Kooi (SIS mixers), M. Thielman, K. Hickerson, and J. Kooi (receiver electronics), W. Schaal (structural design). N. Erickson at U. Massachussets is responsible for the local oscillator systems. A. Harris at U. Maryland is developing the wideband analog correlation spectrometer backend. D. Hawkins at OVRO is developing 
the digital correlation spectrometer technology. At JPL, the receiver group led by W. R. McGrath will develop the HEB channels, while the superconducting device group led by H. G. LeDuc fabricates both the SIS and HEB mixers. The CASIMIR science team includes: G. Blake, J. Keene, and T. Phillips (Caltech); Paul Goldsmith (Cornell); Bill Langer (JPL); Mark Morris (UCLA); and Andy Harris (U. Maryland).

The development of CASIMIR is supported by the NASA/USRA SOFIA instrument development program.

\section{REFERENCES}

1. D. A. Neufeld \& M. J. Kaufman, "Radiative Cooling of Warm Molecular Gas", Ap. J., 418, 263, 1993.

2. R. Stark, F. F. S. van der Tak \& E. F. van Dishoeck, "Detection of interstellar $\mathrm{H}_{2} \mathrm{D}^{+}$emission", Ap. J. (Letters), 521, L67-L70, 1999.

3. R. T. Boreiko \& A. L. Betz, "A search for the rotational transitions $\mathrm{H}_{2} \mathrm{D}^{+}$at $1370 \mathrm{GHz}$ and $\mathrm{H}_{3} \mathrm{O}^{+}$at $985 \mathrm{GHz}$ ", Ap. J. (Letters), 405, L39-L42, 1993.

4. C. L. Bennet, D. J.Fixsen, G. Hinshaw, J. C. Mather, S. H. Moseley, E. L. Wright, R. E. Eplee, J. Gales, T. Hewagama, R. B. Isaacman, R. A. Shafer \& K. Turpie, "Morphology of the interstellar cooling lines detected by COBE", Ap. J., 434, 587-598, 1994.

5. R. T. Boreiko \& A. L. Betz, "Heterodyne spectroscopy of the $J=22-21 \mathrm{CO}$ line in Orion", Ap. J. (Letters), 346, L97-L100, 1989.

6. J. Zmuidzinas \& H. G. LeDuc, "Quasi-optical slot antenna SIS mixers", IEEE Trans. Microwave Theory Tech., MTT-40, 1797-1804, 1992.

7. J. Kooi, J. A. Stern, G. Chattopadhyay, H. G. LeDuc, B. Bumble \& J. Zmuidzinas, "Low Loss NbTiN Films for THz SIS Mixer Tuning Circuits", Int J. IR and MM Waves, 19, 373, 1998.

8. J. Kawamura, J. Chen, D. Miller, J. Kooi, J. Zmuidzinas, B. Bumble, H. G LeDuc \& J. A. Stern, "Low-noise submillimeter-wave NbTiN superconducting tunnel junction mixers", Appl. Phys. Lett., 75, 4013-4015, 1999.

9. J. Kooi, private communication, 2000.

10. J. Kawamura, J.,D. Miller, J. Chen, J. Zmuidzinas, B. Bumble, H. G. LeDuc \& J. A. Stern, "Very High Current Density Nb/AlN/Nb Tunnel Junctions for Low-noise Submillimeter Mixers", Appl. Phys. Lett., in press, 2000.

11. B. Bumble \& H. G. LeDuc, "Fabrication of a diffusion cooled superconducting hot electron bolometer for $\mathrm{THz}$ mixing applications", IEEE Trans. Appl. Superconductivity, 7, 3560, 1997.

12. P. J. Burke, R. J. Schoelkopf, D. E. Prober, A. Skalare, W. R. McGrath, B. Bumble \& H. G. LeDuc

"Length scaling of bandwidth and noise in hot-electron superconducting mixers", App. Phys. Lett., 68, 3344, 1996.

13. B. Karasik, M. C. Gaidis, W. R. McGrath, B. Bumble \& H. G. LeDuc, "A low-noise $2.5 \mathrm{THz}$ superconductive Nb hot-electron mixer", IEEE Trans. Appl. Superconductivity, 7, 3580, 1997.

14. Pamtech Inc., 1151 Avenida ACASO, Camirrilo, CA, 93013, USA.

15. Th. de Graauw et al., "Heterodyne instrument for FIRST (HIFI): preliminary design", SPIE, 3357, 336-3347, 1998.

16. CTT Inc., 3005 Democracy Way, Santa Clara, CA, 95054, USA.

17. J. Horn and O. Siebertz, F. Schmulling, C. Kunz, R. Schieder \& G. Winnewisser, "A 4x1 GHz array acoustooptical spectrometer", Experimental Astronomy, 9, 17-38, 1999.

18. R. Güsten, F. Schaefer, P. van der Waal, R. Stark, R. Scheider, U. U. Graf, J. Stustki, H. Röser, H. Hübers \& P. Hartogh, "GREAT: the first generation German heterodyne receiver for SOFIA", SPIE, these proceedings, 2000. 19. D. Hawkins, "COBRA digital correlator system", SPIE., 4015, 2000.

20. A. I. Harris, K. G. Isaak \& J. Zmuidzinas, "WASP: a wideband spectrometer for heterodyne spectroscopy", SPIE, 3357, 384-394, 1998.

21. A. I. Harris \& J. Zmuidzinas, "WASP2: a $4 \mathrm{GHz}$ bandwidth analog correlator spectrometer", SPIE, 4015, 2000. 